\title{
A EPILEPSIA NA NEUROCISTICERCOSE
}

\author{
Luís MARques-Assis * \\ LuIZ ORTIZ F.**
}

Dentre os processos inflamatórios do sistema nervoso central que costumam assumir forma crônica ressalta, pela freqüência com que incide em nosso meio, a neurocisticercose. Entre os quadros clínicos que esta afecção pode determinar, devem ser destacadas as síndromes epilépticas, associadas ou não a outras manifestações. No presente trabalho propomo-nos a estudar, do ponto de vista clínico-eletrencefalográfico, 131 casos de epilepsia com etiologia cisticercótica comprovada.

\section{MATERIAL E MÉTODOS}

Foram estudados pacientes internados ou registrados no Ambulatório da Clínica Neurológica do Departamento de Neuropsiquiatria do Hospital das Clínicas da Faculdade de Medicina da Universidade de São Paulo. O diagnóstíco de neurocisticercose foi firmado mediante a positividade da reação de Weinberg no líquido cefalorraqueano (128 casos) ou mediante necrópsia ( 3 casos); em 8 casos, além da positividade daquela reação, o diagnóstico foi confirmado pela necrópsia.

A idade variou de 2 a 68 anos; 75 pacientes eram do sexo masculino e 56 do sexo feminino; 117 eram brancos, 10 eram pardos, 3 eram pretos e, um, amarelo. A idade de inicio da doença predominou na primeira década (Quadro 1).

\begin{tabular}{ccc}
$\begin{array}{c}\text { Idade de inicio } \\
\text { (anos) }\end{array}$ & Neurocisticercose & Epilepsias em geral \\
\hline $\begin{array}{r}0 \text { a } 9 \\
10 \text { a } 19\end{array}$ & 47 casos $(36 \%)$ & 316 casos $(26 \%)$ \\
20 a 29 & 25 casos $(19 \%)$ & 578 casos $(47 \%)$ \\
30 ou mais & 30 casos $(23 \%)$ & 134 casos $(11 \%)$ \\
T o t a 1 & 131 casos & 1217 casos
\end{tabular}

Quadro 1 - Casos de neurocisticercose em relação às epilepsias em geral no que se refere à idade de intcio.

Trabalho realizado na Clínica Neurológica (Prof. H. M. Canelas) do Departamento de Neuropsiquiatria da Fac. Med. Univ. São Paulo e apresentado no V Congresso Brasileiro de Neurologia e IIr Congresso Brasileiro de Eletrencefalografia e Neurofisiologia Clínica, São Paulo, 12-15 de julho de 1972: * Médico assistente; ** Médico estagiário. 
O estudo foi feito em relação ao tipo de epilepsia, à idade do início, ao tempo decorrido após a primeira crise e à frequência das crises relativamente às epilepsias em geral. Investgação foi feita também quanto às características da cefaléia, quando presente, e quanto aos achados neurológicos, liquóricos, eletrencefalográficos, radiológicos simples e contrastados e anátomo-patológicos. Para maiores detalhes sobre os métodos utilizados, verificar em publicações anteriores ${ }^{2},{ }^{10}$.

\section{R E S U L T A D O S}

Os resultados referentes à idade de início, ao tipo das manifestações epilepticas, à duração da epilepsia e à frequiência das crises, relativamente às epilepsias em geral, podem ser observados nos quadros 1, 2, 3 e 4. A cefaléia estava presente em 81 casos ( $62 \%)$; em 70 assumiu caráter paroxístico, em 8 caráter contínuo, tendo havido concomitância de ambos em três casos. Dos 70 pacientes com cefaléia paroxística, em 47 não havia sinais de hipertensão intracraniana; dos 8 casos com cefaléia continua, em apenas um, esses sinais não estavam presentes. o exame neurológico foi normal em 73 casos $(54 \%)$ e anormal em 58: sinais de hipertensão intracraniana (HIC) foram constatados em 36 casos, amaurose em 9 e sinais focais em 13 casos.

O exame do líquido cefalorraqueano (LCR) foi feito em 130 casos. A pressão liquórica, medida em 116 casos, mostrou-se elevada em 26, sendo os niveis encontrados normais em 90 casos. Contagem de células foi feita em 129 casos, sendo constatada pleiocitose em 105, com eosinofilorraquia em 63 casos. As proteínas liquóricas foram dosadas em 126 casos; em 55 havia hiperproteinorraquia. A reaçāo de Weinberg, específica para a cisticercose, feita no liquor em todos os casos, foi positiva em 128.

O registro eletrencefalográfico foi obtido em 110 casos, tendo sido normal em 37. Dos 73 casos em que foram constatadas anormalidades, estas se revelaram de tipo paroxístico focal em 47, contínuo focal em 10, paroxístico difuso em 6 e contínuo difuso em 10 casos; em dois casos havia concomitância de mais de um tipo de anormalidade.

O exame radiológico simples, realizado em 75 pacientes, foi normal em 40 , revelando sinais de hipertensão intracraniana em 26 e presença de calcificaçōes em 9 casos. A carotidoangiografia, feita em 25 casos, mostrou sinais indiretos de dilatação ventricular em 15, desvios vasculares em 5 e oclusões arteriais em três, sendo normal em dois casos. O pneumencefalograma, feito em 6 casos, mostrou sinais de atrofia em dois, de processo expansivo em um, de hidrocefalia em dois e de processo quiasmático em um caso. Exame iodoventriculográfico, feito em 6 casos, mostrou bloqueio parcial ou total ao nivel do aqueduto e quarto ventriculo em 4 casos e dilataçăo ventricular em um caso; em um caso o exame foi inconclusivo.

o exame necroscópico, feito em 11 casos, confirmou a etiologla cisticercótica da doença em dois casos com reação de Weinberg negativa no liquor e em um caso que não foi submetido a punção liquórica. Em 8 casos mostrou a presença de cisticercos cerebrais ou intradurais.

\section{O M E N T A R I O S}

Sendo a cisticercose do sistema nervoso central bastante freqüente nos povos menos desenvolvidos, sua incidência é elevada em nosso meio. Por outro lado, a neurocisticercose pode se exteriorizar clinicamente de forma aguda, subaguda ou crônica; nas duas últimas eventualidades, com freqüência existem manifestações de natureza epiléptica, que podem se constituir no único sintoma da doenca. Embora sendo etiologia relativamente 
rara ${ }^{11}$ a cisticercose tem sido valorizada como sendo epileptogênica por diversas autores $1,2,5,6,7,13,14$.

Canelas $^{2}$ (1962) fez extensa revisão sobre a neurocisticercose em nosso meio, encontrando epilepsia em grande parte dos casos $(46,6 \%)$, em proporção maior que os sinais de HIC $(35,9 \%)$ e que as alterações neurológicas focais ou difusas $(24,5 \%)$. No que se refere ao tipo das manifestações epilépticas, no grupo em que as crises ocorreram sem sinais neurológicos concomitantes, predominaram as formas convulsivas, sendo encontradas em proporção menor as crises de tipo psicomotor; 65\% dos casos apresentaram também sinais de hipertensão intracraniana. Brotto ${ }^{1}$, em 1947, já havia chamado a atenção para o fato de serem as formas epilépticas as mais freqüentemente encontradas na neurocisticercose.

Dentre as formas raras de epilepsia na neurocisticercose foram descritas as psicomotoras (Trelles ${ }^{15}$ ). Também a presença de epilepsia tem sido referida nas formas hemiplégicas da doença ${ }^{3}$ e, embora em menor grau, nas formas do ângulo pontocerebelar ${ }^{4}$.

Em nossos casos, no que diz respeito à idade de início da doença, quando comparado o grupo de neurocisticercose com o das epilepsias em geral (quadro 1), verifica-se maior incidência na primeira década da vida $\mathrm{e}$ a partir da quarta década no primeiro grupo em relação às epilepsias em geral; nestas a doença se inicia predominantemente na segunda década $(47 \%$ dos casos).

Quanto ao tipo da epilepsia, em nosso material predominaram nitidamente as formas convulsivas da epilepsia, sendo as mais raras as de tipo bravais-jacksoniana sem generalização (referidas em apenas dois casos); em $8 \%$ dos casos foram descritas crises psicomotoras; porém, em apenas 5 casos as crises de automatismo ocorreram isoladamente. Relativamente às epilepsias em geral (quadro 2) verifica-se incidência menor das convulsões generalizadas na neurocisticercose.

\begin{tabular}{lrr}
\hline Crises & $\begin{array}{c}\text { Neurocisti- } \\
\text { cercose }\end{array}$ & $\begin{array}{c}\text { Epilepsias em } \\
\text { geral }\end{array}$ \\
\hline \hline Convulsivas & 80 casos $(61 \%)$ & 1142 casos $(94 \%)$ \\
Psicomotoras & 11 casos $(8 \%)$ & 90 casos $(8 \%)$ \\
Bravais-jacksonianas & 6 casos $(4 \%)$ & 86 casos $(7 \%)$ \\
Outros tipos & 41 casos $(31 \%)$ & 53 casos $(4 \%)$
\end{tabular}

\begin{tabular}{c}
\hline Quadro 2 - Casos de neurocisticercose em relacão às epi- \\
lepsias em geral no que se refere às mani- \\
festaçoes clínicas.
\end{tabular}

No que se refere à duração da epilepsia, comparados os casos de neurocisticercose com as epilepsias em geral (quadro 3) nota-se, como era de se esperar, predomínio das formas com duração de um ano ou menos no primeiro grupo, sendo mais raras as formas com 3 anos ou mais de evolução. 


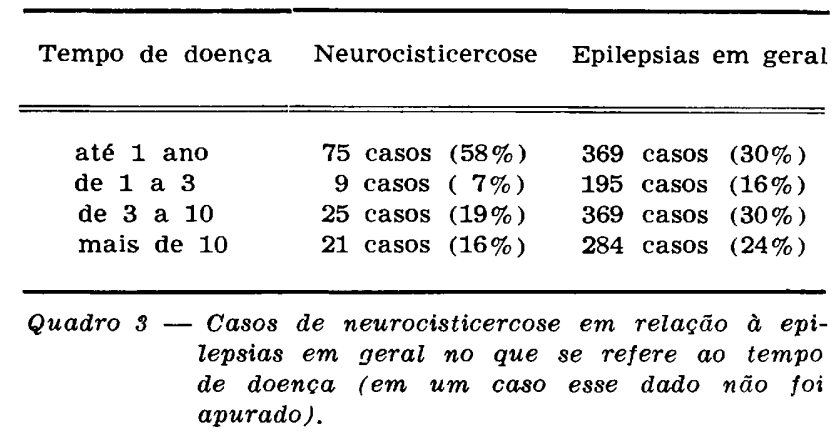

A severidade da doença, traduzida pela frequência das crises, relativamente às epilepsias em geral (quadro 4) torna-se de interpretação difícil levando em conta a alta porcentagem de casos nos quais esse dado é ignorado no primeiro grupo. No entanto, o estudo percentual comparativo, sendo desprezados os 45 casos em que a freqüência das crises é ignorada, permite constatar maior benignidade no grupo da neurocisticercose, com menor número de casos com freqüência mensal ou com mais de uma crise por mês, relativamente às epilepsias em geral. Em virtude da própria natureza da doença nota-se também maior número das formas iniciais das epilepsias no grupo da cisticercose (casos que procuram atendimento médico imediatamente após as primeiras crises).

\begin{tabular}{|c|c|c|}
\hline Freqüência & Neurocisticercose & Epilepsias em geral \\
\hline até $7 / 7$ dias & $14 \operatorname{casos}(16 \%)$ & 309 casos $(25 \%)$ \\
\hline de $8 / 8$ a $30 / 30$ & 21 casos $(24 \%)$ & 361 casos $(30 \%)$ \\
\hline de $31 / 31$ a $90 / 90$ & 16 casos $(18 \%)$ & 147 casos $(12 \%)$ \\
\hline inferior a $\mathbf{9 0 / 9 0}$ & $11 \operatorname{casos}(13 \%)$ & 197 casos $(16 \%)$ \\
\hline formas iniciais & $24 \operatorname{casos}(29 \%)$ & 213 casos $(17 \%)$ \\
\hline $\begin{array}{r}\text { Quadro } 4-\text { Casos } \\
\text { lepsias } \\
\text { das cr } \\
\text { apurad }\end{array}$ & $\begin{array}{l}\text { de neurocisticercose } \\
\text { em geral no que se } \\
\text { ises rem } 45 \text { casos } \\
\text { o). }\end{array}$ & $\begin{array}{l}\text { em relação às epi- } \\
\text { refere à freqüência } \\
\text { esse dado näo foi }\end{array}$ \\
\hline
\end{tabular}

A cefaléia está entre os sintomas comumente referidos na neurocisticercose e, não raramente, faz parte das queixas dos pacientes epilépticos de modo geral. No grupo estudado, esse sintoma estava presente na maior parte dos casos $(68 \%)$, assumindo caráter paroxístico $(78 \%)$ ou contínuo $(16 \%)$. Cumpre notar que em 47 casos com cefaléia paroxística $(67 \%)$ não havia concomitantemente sinais de hipertensão intracraniana; por outro lado, em apenas dois casos com cefaléia contínua (13\%) esses sinais não estavam presentes. Tendn em vista esses dados, pode-se concluir 
que a presença de crises de cefaléias em paciente com neurocisticercose não implica em aumento da pressão intracraniana, sendo diversa sua etiopatogenia.

Canelas $^{2}$ e Pupo ${ }^{14}$ colocam as formas hipertensivas da neurocisticercose como as mais freqüentes depois das formas convulsivas. Em nosso material, em que foram estudados casos com alguma forma de epilepsia, a hipertensão intracraniana foi constatada em 36 casos (26\%); em 8 casos havia amaurose, em 5 dos quais não havia HIC atual, o que faz supor de sua existência prévia. Por outro lado, sinais neurológicos focais foram encontrados em apenas 13 casos $(10 \%)$. Portanto, os casos que apresentaram apenas manifestaçōes epilépticas, sem sinais neurológicos ou de HIC, constituiram a maior parte (62\%) e devem corresponder ao contingente ambulatorial do material estudado.

No que diz respeito ao traçado eletrencefalográfico, Longo e col. ${ }^{8}$, estudando as cisticercoses encefálicas em geral, encontraram raramente traçados normais, chamando a atenção para o valor das disritmias paroxísticas como indicativas da presença de convulsões no quadro. Lombardo e Mateos ${ }^{7}$ ressaltaram a prevalência das disritmias difusas em relação às focais. Canedas ${ }^{2}$, analisando a neurocisticercose em geral, encontrou $30 \%$ dos casos com EEG normal e predomínio das anormalidades localizadas em relação à difusas; quando analisadas apenas as formas epilépticas da doença, o índice de normalidade foi maior $(44 \%)$, sendo o comportamento semelhante no grupo com EEG anormal. Em nossos casos os resultados foram semelhantes: estudo comparativo com as epilepsias em geral (quadro 5), excluídos os casos com disritmia contínua, mostra que na neurocisticercose os traçados normais foram encontrados em maiores índices percentuais.

\begin{tabular}{lrr}
\hline EEG & Neurocisticercose & Epilepsias em geral \\
\hline \hline Normal & 37 casos $(41 \%)$ & 367 casos $(30 \%)$ \\
Focal & 47 casos $(52 \%)$ & 699 casos $(58 \%)$ \\
Difuso & 6 casos $(7 \%)$ & 151 casos $(12 \%)$
\end{tabular}

\begin{tabular}{c}
\hline Quadro $5-$ o eletrencefalograma na neurocisticercose (90 \\
casos com disritmia paroxistica) relativamente \\
às exilepsias em geral.
\end{tabular}

Os achados do presente trabalho no que se refere ao tempo de doença, à severidade da epilepsia e ao eletrencefalograma corroboram os referidos por Marques-Assis e Morais $\mathrm{Jr},{ }^{12}$ que estudaram as epilepsias em pacientes com liquido cefalorraqueano alterado, inclusive com meningencefalites específicas (cisticercóticas e luéticas).

Os exames radiológicos simples e contrastados, realizados nos casos em que investigação mais profunda se tornou necessária, comprovou, em alguns deles, a existência de alterações encefálicas de natureza neurocirúrgica, formas essas já descritas ıa doença. 
Dos 11 casos que foram submetidos à autópsia, em dois a reação de Weinberg havia sido negativa, mas havia pleiocitose e eosinofilorraquia. Esse achado assume importância e nunca é demais repetir que, para a feitura do diagnóstico de neurocisticercose não é indispensável a positividade daquela reação, ficando também demonstrado o valor que deve ser atribuído à presença da eosinofilorraquia nessas condições.

\section{RESUMO E CONCLUSOES}

Foram estudados 131 casos de epilepsia com etiologia cisticercótica comprovada. A idade variou de 2 a 68 anos; 75 pacientes eram de sexo masculino e. 56 de sexo feminino; 117 eram brancos, 10 pardos, três pretos e um amarelo. A idade de início da doença predominou na primeira década.

O estudo foi feito em relação ao tipo de epilepsia, ao tempo decorrido após a primeira crise, à freqüência das crises e ao eletrencefalograma relativamente às epilepsias em geral. Investigação foi feita também em relação à cefaléia, aos achados neurológicos, liquóricos, radiológicos simples e contrastados e anátomo-patológicos.

A análise dos resultados permitiu aos autores as seguintes conclusões: 1) No grupo de neurocisticercose o início da doença predominou na primeira e a partir da quarta década em relação às epilepsias em geral. 2) Dentre as manifestaçōes clínicas predominaram as formas convulsivas da doença $(61 \%)$, sendo as crises bravas-jacksonianas as menos freqüentes (4\%); quando comparadas com as epilepsias em geral, verifica-se incidência menor das convulsões generalizadas na neurocisticercose. 3) Quanto ao tempo de doença, verifica-se predomínio das formas de duração mais curta (um ano ou menos) na neurocisticercose. 4) A severidade da epilepsia, traduzida pela freqüência das crises, foi menor no grupo com neurocisticercose. 5) Excluídos os casos com anormalidades eletrencefalográficas contínuas, foram encontrados maiores índices percentuais de EEG normal na neurocisticercose que nas epilepsias em geral. 6) A cefaléia estava presente em $68 \%$ dos casos, assumindo caráter paroxístico na maior parte $(78 \%)$; em $67 \%$ dos casos com cefaléia paroxística não havia concomitantemente sinais de hipertensão intracraniana; nos casos com cefaléia contínua, em apenas dois (13\%) esses sinais não estavam presentes. 7) $\mathrm{Na}$ maior parte dos casos $(62 \%)$ as manifestações epilépticas se apresentaram isoladamente, sem sinais neurológicos focais ou sinais de hipertensão intracraniana.

\section{S U M M A R Y}

\section{The epilepsy in neurocysticercosis}

One hundred and thirty one cases of epilepsy with neurocysticercosis were studied. The investigation was made in regard to the type of epilepsy, the disease duration, the frequency of seizures and the electroencephalographic pattern regarding the epilepsies in general. The study was also 
done concerning the headache, the neurological findings, the cerebrospinal fluid, plain and contrasted radiologic examination and histopathology.

The author draw the following conclusions: 1) In the neurocysticercosis group the onset of disease predominate in the first decada and from the fourth decada. 2) Concerning the type of seizures, the convulsions predominate; the Jacksonian fits were the less frequent. 3) Concerning the duration of disease the epilepsy with short duration (one year or less) predominate in the neurocysticercosis group. 4) The severity of epilepsy (frequency of seizures) was smaller in the neurocysticercosis group. 5) Excluding the cases with continuous EEG abnormalities, there were more normal EEG in neurocysticercosis group than in epilepsies in general. 6) The headache was present in $68 \%$ of cases, with paroxysmal pattern in the greater number $(78 \%)$; in $67 \%$ of cases with paroxysmal headache the intracranial hypertension signs were absent; in the cases with continuous headache these signs were not present in only two cases $(13 \%)$. 7) In the greater number $(62 \%)$ the patients have presented only epileptic manifestations without focal neurological signs or intracranial hypertension.

\section{R E F E R E N I A S}

1. BRotto, W. - Aspectos neurológicos da cisticercose. Arq. Neuro-Psiquiat. (São Paulo) 5:258, 1947.

2. CANElAS, H. M. - Neurocisticercose: incidência, diagnóstico e formas clínicas. Arq. Neuro-Psiquiat. (São Paulo) 20:1, 1962.

3. CANElAS, H. M. \& CRIJZ, O. R. - Neurocisticercose: formas clinicas pouco frequientes. I - Formas hemiplégicas. Arq. Neuro-Psiquiat. (São Paulo) 20: 89, 1962.

4. CANElAS, H. M.; CRUZ, O. R. \& TENUTO, R. - Neurocisticercose: formas clínicas pouco freqüentes. II - Formas do ângulo ponto-cerebelar. Arq. Neuro-Psiquiat. (São Paulo) 20:102, 1962.

5. FU YA-KO. - Clinical analysis of 416 cases of epilepsy. Clin. J. Neurol. Psychiat. 2:173, 1956. Resumo em Epilepsy Abstr, 1. ${ }^{\text {a }}$ Parte, n.o 1605, 1947/1967.

6. HEINZ, H. J. \& KLINTWORTH, G. K. - Cysticercosis in the aetiology of eptlepsy. S. Afr. J. med. Sci. 30:32, 1956. Resumo em Epilepsy Abstr., 1.a Parte, n.o 2306, 1947/1967.

7. LOMBARDO, L. \& MATEOS, J. H. - Cerebral cysticercosis in Mexico. Neurology (Minneapolis) 11:824, 1961.

8. LONGO, P. W.; PUPO, P. P.; ZUKERMAN, E.; LONGO, R. H.; MOREIRA, M. H. F. R.; JORDY, C.; LIMA, J. G. C. \& ZORLINI, G. - Aspectos eletrencefalográficos da cisticercose encefálica. Arq Neuro-Psiquiat. (São Paulo) 17: $357,1959$.

9. MARQUES-ASSIS, L. - Tratamento medicamentoso de 1217 pacientes epilépticos: estudo em relacão ao tipo de epilepsia e ao eletrencelograma. Arq. Neuro-Psiquiat. (São Paulo) 27:312, 1569.

10. MARQUES-ASSIS, L. - Tratamento medicamentoso de 1217 pacientes epilépticos: estudo em relação à idade de inicio, ao tempo de doença e à freqüência das crises. Arq. Neuro-Psiquiat. (São Paulo) 28:44, 1970.

11. MARQUES-ASSIS, L. \& ABDALlA, H. - O liquido cefalorraqueano nas epilepsias. Arq. Neuro-Psiquiat. (São Faulo) 21:279, 1963.

12. MARQUES-ASSIS, L. \& MORAIS Jr., L. C. - Estudo clínico de 84 pacientes epilépticos com liquido cefalorraqueano alterado. Arq. Neuro-Psiquiat. (São Paulo) 29:55, 1971. 
13. PAWELL, S. J.; PROCTOR, E. M.; WILMOT, A. J. \& MAC LEOD, I. N. Cysticercosis and epilepsy in Africans: a clinical and serologic study. Ann. trop. Med. Parasit. 60:152, 1966. Resumo em Epilepsy Abstr., 1. ${ }^{\mathrm{a}}$ Parte, n.o 2382, 1947/1967.

14. PUPO, P. P. - Cysticercosis of the nervous system. Rev. Neuro-Psiquiat. (Lima) 27:70, 1964.

15. TRELLES, J. O. \& ROEDENBERG, S. D. - Estudios sobre neurocisticercosis. III - Formas clinicas poco frecuentes de cisticercosis. Rev. Neuro-Psiquiat. (LIma) 17:15, 1954.

Clínica Neurológica - Faculdade de Medicina da Universidade de São Paulo - Caixa Postal $\$ 461$ - 01000 São Paulo, SP - Brasil. 